\title{
Characteristics of Neuropsychiatric Mobile Health Trials: Cross-Sectional Analysis of Studies Registered on ClinicalTrials.gov
}

Mia Tova Minen ${ }^{1}$, MD, MPH; Julia Frederica Reichel², BA; Pallavi Pemmireddy ${ }^{2}$, BA; Elizabeth Loder ${ }^{3}$, MD, MPH; John Torous ${ }^{4}$, MD, MBI

\author{
${ }^{1}$ NYU Langone Health, New York, NY, United States \\ ${ }^{2}$ Barnard College of Columbia University, New York, NY, United States \\ ${ }^{3}$ Brigham and Women's Hospital, Boston, MA, United States \\ ${ }^{4}$ Beth Israel Deaconess Medical Center, Brookline, MA, United States
}

\section{Corresponding Author:}

Mia Tova Minen, MD, MPH

NYU Langone Health

222 E 41st Street,

New York, NY, 10017

United States

Phone: 12122637744

Email: minenmd@gmail.com

\begin{abstract}
Background: The development of mobile health (mHealth) technologies is progressing at a faster pace than that of the science to evaluate their validity and efficacy. Under the International Committee of Journal Medical Editors (ICMJE) guidelines, clinical trials that prospectively assign people to interventions should be registered with a database before the initiation of the study.

Objective: The aim of this study was to better understand the smartphone mHealth trials for high-burden neuropsychiatric conditions registered on ClinicalTrials.gov through November 2018, including the number, types, and characteristics of the studies being conducted; the frequency and timing of any outcome changes; and the reporting of results.

Methods: We conducted a systematic search of ClinicalTrials.gov for the top 10 most disabling neuropsychiatric conditions and prespecified terms related to mHealth. According to the 2016 World Health Organization Global Burden of Disease Study, the top 10 most disabling neuropsychiatric conditions are (1) stroke, (2) migraine, (3) major depressive disorder, (4) Alzheimer disease and other dementias, (5) anxiety disorders, (6) alcohol use disorders, (7) opioid use disorders, (8) epilepsy, (9) schizophrenia, and (10) other mental and substance use disorders. There were no date, location, or status restrictions.

Results: Our search identified 135 studies. A total of 28.9\% (39/135) of studies evaluated interventions for major depressive disorder, $14.1 \%$ (19/135) of studies evaluated interventions for alcohol use disorders, $12.6 \%$ (17/135) of studies evaluated interventions for stroke, $11.1 \%(15 / 135)$ of studies evaluated interventions for schizophrenia, $8.1 \%$ (11/135) of studies evaluated interventions for anxiety disorders, $8.1 \%$ (11/135) of studies evaluated interventions for other mental and substance use disorders, $7.4 \%(10 / 135)$ of studies evaluated interventions for opioid use disorders, $3.7 \%(5 / 135)$ of studies evaluated interventions for Alzheimer disease or other dementias, 3.0\% (4/135) of studies evaluated interventions for epilepsy, and 3.0\% (4/135) of studies evaluated interventions for migraine. The studies were first registered in 2008; more than half of the studies were registered from 2016 to 2018. A total of $18.5 \%$ (25/135) of trials had results reported in some publicly accessible location. Across all the studies, the mean estimated enrollment (reported by the study) was 1078, although the median was only 100. In addition, across all the studies, the actual reported enrollment was lower, with a mean of 249 and a median of 80 . Only about a quarter of the studies $(35 / 135,25.9 \%)$ were funded by the National Institutes of Health.
\end{abstract}

Conclusions: Despite the increasing use of health-based technologies, this analysis of ClinicalTrials.gov suggests that only a few apps for high-burden neuropsychiatric conditions are being clinically evaluated in trials.

(JMIR Mhealth Uhealth 2020;8(8):e16180) doi: $\underline{10.2196 / 16180}$ 


\section{KEYWORDS}

smartphones; mobile phones; apps; mental health; regulation; stroke; migraine; major depressive disorder; Alzheimer disease; anxiety disorders; alcohol use disorders; opioid use disorders; epilepsy; schizophrenia

\section{Introduction}

The field of mobile health (mHealth), broadly defined as health care interventions that are delivered by mobile devices such as smartphones and tablets, is growing rapidly. Currently, there are over 325,000 mHealth apps [1], and the field of mHealth continues to attract new market entrants ( $28 \%$ of digital health practitioners have less than 2 years of industry experience) [1]. In addition, the global mHealth app market size is expected to hit 236 billion by 2026 [2]. mHealth apps claim to have varied purposes, from improving treatment adherence to increasing physical activity, to supplementing in-person counseling, and much more. The development of mHealth technologies is currently progressing at a much faster pace than that of the science to evaluate their validity and efficacy [3]. Thus, it is possible that ineffective or even harmful mHealth technologies might enter clinical practice without adequate evaluation [4]. For example, many apps make scientific claims on the app stores, but less than $2 \%$ of the apps can offer clinical evidence to back such claims [5]. The topic of digital health regulation by the Food and Drug Administration in the United States has been a news story since the fall of 2019 when it was discussed by a presidential candidate.

Currently, one path to increase scientific validity and transparency in the digital health space is through the mechanisms in place around publication. Since 2005, a condition of consideration for publication in the International Committee of Journal Medical Editors (ICJME) has been prospective registration of clinical trials. The ICJME defines a clinical trial as "any research project that prospectively assigns people or a group of people to an intervention, with or without concurrent comparison or control groups, to study the relationship between a health-related intervention and a health outcome." Trial registration is supposed to occur "in a public trials registry at or before the time of first patient enrollment." Registration requirements are intended to prevent well-documented problems that arise when the results of the trials are either unreported or are selectively reported [6]. A majority of the clinical trials are registered at ClinicalTrials.gov, a division of the US National Library of Medicine [7]. It is also possible to post trial results on ClinicalTrials.gov, although such posting is only required for certain studies. When reporting requirements apply, the results must generally be posted no later than 1 year after final data collection for the primary outcome [8].

Previous examinations of ClinicalTrials.gov have provided details on the types of studies being conducted in various fields and the various aspects of such studies, for example, location, number of study participants, interventions, and funders $[9,10]$. As is the case with clinical trials, in general, it is likely that many trials of mHealth interventions are not compliant with the requirements for trial registration. Selective reporting of results is of special concern in mHealth studies as they often collect vast amounts of data from surveys and sensors-often millions of data points from smartphone sensors and wearables. This creates many opportunities for both intentional and inadvertent systematic errors or biases in ongoing data collection, analysis, and interpretation. Prospective trial registration is an important protection against such mistakes, as well as a safeguard against intentional abuse.

However, the characteristics, number, and quality of registered trials of mHealth interventions for most therapeutic areas, including neuropsychiatric disorders, are unknown. According to the 2016 World Health Organization (WHO) Global Burden of Disease Study [11], the top 10 most disabling noninfectious neuropsychiatric conditions are, in the following order of ranking, (1) stroke, (2) migraine, (3) major depressive disorder, (4) Alzheimer disease and other dementias, (5) anxiety disorders, (6) opioid use disorders, (7) alcohol use disorders, (8) epilepsy, (9) schizophrenia, and (10) other mental and substance use disorders. For these conditions, we sought to evaluate the (1) number of mHealth trials of smartphone interventions registered on ClinicalTrials.gov; (2) study characteristics, for example, location of the study, type of interventions studied, number of study participants, length of study, and funder (National Institutes of Health [NIH] or other), among other characteristics; (3) frequency and timing of any outcome changes in the trial registry; and (4) the proportion of such studies that had reported results on ClinicalTrials.gov or in journal publications. Neuropsychiatric disorders comprise a broad range of medical conditions involving neurologic or psychiatric disturbances, including mental and behavioral disorders. Globally, they are the third leading cause of disability-adjusted life years (DALYs) $[12,13]$. Neuropsychiatric conditions are likely to be particularly amenable to mHealth interventions as their treatment may involve behavioral therapies and efforts to improve medication adherence, and there is a great need for scalable and accessible interventions [14-16].

\section{Methods}

According to the 2016 WHO Global Burden of Disease Study, [11] the following comprised the top 10 most disabling neuropsychiatric conditions worldwide: (1) stroke, (2) migraine, (3) major depressive disorder, (4) Alzheimer disease and other dementias, (5) anxiety disorders, (6) alcohol use disorders, (7) opioid use disorders, (8) epilepsy, (9) schizophrenia, and (10) other mental and substance use disorders (Multimedia Appendix 1 [17-149]). These conditions were searched for in ClinicalTrials.gov, along with smartphone-based keyword terms, and data were abstracted accordingly.

Data were summarized descriptively. Of note, we examined the reporting of results using two different methods. In the first method, for studies registered 3 or more years prior, we examined how many of these studies reported results either via one or both of the following: results reported on ClinicalTrials.gov or results automatically indexed to ClinicalTrials.gov. In the second method, we examined the studies marked completed on ClinicalTrials.gov by October 
2017 (1 year before the date of data abstraction) and then examined which of those studies reported results either on ClinicalTrials.gov and/or had results automatically indexed to ClinicalTrials.gov.

We also analyzed whether there were any associations among (1) the number of study participants and study completion status, (2) the number of study participants and study results reporting status (3), the length of study intervention and completion status, and (4) the length of study intervention and results reporting status. We utilized $t$ tests and regression models to analyze the results. A statistical analysis was conducted in the $R$ programming environment (version R 3.6.1; The R Foundation).

Per the self-documentation form from the New York University (NYU) School of Medicine (SOM), the research did not involve human subjects. Thus, consistent with the NYU SOM institutional review board (IRB) policy and federal regulations governing human subject research, an IRB review was not required.

\section{Results}

A total of 135 studies on ClinicalTrials.gov met the search criteria. As shown in Multimedia Appendix 2 [17-64,66-82,84-114,116-133,135-141,143-153], the number of studies registered for each neuropsychiatric condition that used a smartphone for an intervention was as follows: major depressive disorder $(n=39)$, alcohol use disorders $(n=19)$, stroke $(n=17)$, schizophrenia $(n=15)$, anxiety disorders $(n=11)$, other mental and substance use disorders $(\mathrm{n}=11)$, opioid use disorders $(n=10)$, Alzheimer disease and other dementias $(n=5)$, epilepsy $(n=4)$, and migraine $(n=4)$. Multimedia Appendix 1 shows the key findings for the various apps, including the myriad of purposes they served. They ranged from promoting rehabilitation and diet to medication adherence, symptom tracking, cognitive behavioral therapy, and more.

A breakdown of the studies is provided in Table 1. The altered outcomes have been described in Table 2 . 
Table 1. Breakdown of study status, results reporting, location of studies and National Institutes of Health funding.

\begin{tabular}{|c|c|c|c|c|c|c|c|c|c|c|c|}
\hline Characteristics $^{\mathrm{a}, \mathrm{b}}$ & $\begin{array}{l}\text { All condi- } \\
\text { tions }\end{array}$ & Stroke & $\begin{array}{l}\text { Mi- } \\
\text { graine }\end{array}$ & $\begin{array}{l}\text { Major de- } \\
\text { pressive } \\
\text { disorders }\end{array}$ & $\begin{array}{l}\text { Alzheimer } \\
\text { disease } \\
\text { and other } \\
\text { demen- } \\
\text { tias }\end{array}$ & $\begin{array}{l}\text { Anxiety } \\
\text { disorders }\end{array}$ & $\begin{array}{l}\text { Alcohol } \\
\text { use disor- } \\
\text { ders }\end{array}$ & $\begin{array}{l}\text { Opioid } \\
\text { use dis- } \\
\text { orders }\end{array}$ & Epilepsy & $\begin{array}{l}\text { Schizophre- } \\
\text { nia }\end{array}$ & $\begin{array}{l}\text { Other } \\
\text { mental } \\
\text { and sub- } \\
\text { stance } \\
\text { use disor- } \\
\text { ders }\end{array}$ \\
\hline $\begin{array}{l}\text { Number of apps, } \\
\mathrm{n}\end{array}$ & 135 & 17 & 4 & 39 & 5 & 11 & 19 & 10 & 4 & 15 & 11 \\
\hline \multicolumn{12}{|l|}{ Status of study, $n$} \\
\hline $\begin{array}{l}\text { Not yet re- } \\
\text { cruiting }\end{array}$ & 19 & 0 & 2 & 5 & 3 & 0 & 1 & 4 & 0 & 2 & 2 \\
\hline Recruiting & 40 & 8 & 2 & 9 & 1 & 2 & 7 & 3 & 2 & 3 & 3 \\
\hline $\begin{array}{l}\text { Enrolling by } \\
\text { invitation }\end{array}$ & 7 & 1 & 0 & 0 & 1 & 2 & 1 & 0 & 1 & 0 & 1 \\
\hline $\begin{array}{l}\text { Active, not } \\
\text { recruiting }\end{array}$ & 7 & 1 & 0 & 4 & 0 & 0 & 1 & 0 & 0 & 1 & 0 \\
\hline Completed & 59 & 7 & 0 & 21 & 0 & 8 & 7 & 3 & 1 & 9 & 4 \\
\hline Unknown & 3 & 0 & 0 & 0 & 0 & 0 & 2 & 0 & 0 & 0 & 1 \\
\hline
\end{tabular}

Number of participants estimated

$\begin{array}{llllllllllll}\text { Mean (SD) } & 1078.64 & 222.06 & 131 & 199.74 & 196 & 629 & 439.59 & 144.8 & 25066.25 & 102(92.31) & 234.10 \\ & (9088.35) & (470.64) & (52.57) & (347.83) & (175.23) & (1640.23) & (866.02) & (205.22) & (49955.84) & & (241.73) \\ \begin{array}{l}\text { Lowest; } \\ \text { highest, } \mathrm{n}\end{array} & 8 ; & 8 ; 2000 & 90 ; 200 & 15 ; 2000 & 52,448 & 8 ; 5000 & 30 ; 3600 & 9 ; 600 & 65 ; & 36 ; 260 & 40 ; 800 \\ \begin{array}{l}\text { Median } \\ \text { (IQR) }\end{array} & 100,000 & & & & & & & & 100,000 & \\ \end{array}$

\section{Number of participants actual}

\begin{tabular}{|c|c|c|c|c|c|c|c|c|c|c|c|}
\hline Mean (SD) & $\begin{array}{l}242.19 \\
(586.02)\end{array}$ & $\begin{array}{l}301.55 \\
(657.80)\end{array}$ & $\mathrm{N} / \mathrm{A}^{\mathrm{c}}$ & $\begin{array}{l}208.38 \\
(426.21)\end{array}$ & 20 (N/A) & $\begin{array}{l}51.4 \\
(41.52)\end{array}$ & $\begin{array}{l}683.71 \\
(1356.83)\end{array}$ & $\begin{array}{l}67 \\
(79.20)\end{array}$ & 95 (N/A) & $92.3(75.69)$ & $\begin{array}{l}322.33 \\
(123.44)\end{array}$ \\
\hline $\begin{array}{l}\text { Lowest; } \\
\text { highest, n }\end{array}$ & $10 ; 3702$ & $\begin{array}{l}20 \\
2274\end{array}$ & N/A & $11 ; 2010$ & $20 ; 20$ & $10 ; 105$ & $15 ; 3702$ & $11 ; 123$ & $95 ; 95$ & $27 ; 255$ & $180 ; 400$ \\
\hline $\begin{array}{l}\text { Median } \\
\text { (IQR) }\end{array}$ & $\begin{array}{l}80 \\
(137.25)\end{array}$ & $\begin{array}{l}100 \\
(145)\end{array}$ & N/A & $\begin{array}{l}84.5 \\
(105)\end{array}$ & $20(0)$ & $40(66)$ & $\begin{array}{l}58 \\
(395.5)\end{array}$ & $67(56)$ & $95(0)$ & 59.5 (91.25) & 387 (110) \\
\hline
\end{tabular}

Number of days of intervention

$\begin{array}{llllllllllll}\text { Mean (SD) } & 149.26 & 155.88 & 173.75 & 104.44 & 202.2 & 198.91 & 125.90 & 158.6 & 112.25 & 189.93 & 205.18 \\ & (199.76) & (137.44) & (138.29) & (92.29) & (241.06) & (540.84) & (114.42) & (227.36) & (47.282) & (166.73) & (145.18) \\ \begin{array}{l}\text { Lowest; } \\ \text { highest, } \mathrm{n}\end{array} & \begin{array}{l}0.0083 ; \\ 1825\end{array} & 1 ; 456 & 56 ; 365 & 1 ; 395 & 28 ; 548 & 0.00 ; & 7 ; 365 & 7 ; 730 & 84 ; 183 & 30 ; 548 & 30 ; 548 \\ \begin{array}{l}\text { Median } \\ \text { (IQR) }\end{array} & 84(127) & 91(187) & \begin{array}{l}137 \\ (146.25)\end{array} & 84(66) & 42(337) & 14(76) & 84(78.5) & 56 & 91 & 168(95.5) & 183 \\ & & & & & & (121.5) & (24.75) & 1825 & & (80.5)\end{array}$

\section{Regional distribution, $\mathbf{n}$}

$\begin{array}{llllllllllll}\text { Africa } & 1 & 1 & 0 & 0 & 0 & 0 & 0 & 0 & 0 & 0 & 0 \\ \begin{array}{l}\text { Asia and Pa- } \\ \text { cific }\end{array} & 8 & 3 & 0 & 0 & 1 & 0 & 3 & 0 & 0 & 1 & 0 \\ \begin{array}{l}\text { Cen- } \\ \text { tral+South }\end{array} & 5 & 0 & 0 & 4 & 0 & 1 & 0 & 0 & 0 & 0 \\ \begin{array}{l}\text { America } \\ \text { Europe }\end{array} & 28 & 6 & 0 & 7 & 1 & 2 & 5 & 0 & 1 & 4 & 2 \\ \begin{array}{l}\text { Middle East } \\ \text { North Ameri- }\end{array} & 2 & 0 & 0 & 0 & 0 & 1 & 0 & 0 & 0 & 1 & \\ \text { ca } & 7 & 4 & 30 & 3 & 7 & 11 & 10 & 4 & 11 & 9\end{array}$




\begin{tabular}{|c|c|c|c|c|c|c|c|c|c|c|c|}
\hline Characteristics $^{\mathrm{a}, \mathrm{b}}$ & $\begin{array}{l}\text { All condi- } \\
\text { tions }\end{array}$ & Stroke & $\begin{array}{l}\text { Mi- } \\
\text { graine }\end{array}$ & $\begin{array}{l}\text { Major de- } \\
\text { pressive } \\
\text { disorders }\end{array}$ & $\begin{array}{l}\text { Alzheimer } \\
\text { disease } \\
\text { and other } \\
\text { demen- } \\
\text { tias }\end{array}$ & $\begin{array}{l}\text { Anxiety } \\
\text { disorders }\end{array}$ & $\begin{array}{l}\text { Alcohol } \\
\text { use disor- } \\
\text { ders }\end{array}$ & $\begin{array}{l}\text { Opioid } \\
\text { use dis- } \\
\text { orders }\end{array}$ & Epilepsy & $\begin{array}{l}\text { Schizophre- } \\
\text { nia }\end{array}$ & $\begin{array}{l}\text { Other } \\
\text { mental } \\
\text { and sub- } \\
\text { stance } \\
\text { use disor- } \\
\text { ders }\end{array}$ \\
\hline $\begin{array}{l}\text { Study marked as } \\
\text { National Insti- } \\
\text { tutes of Health } \\
\text { funded, } n\end{array}$ & 35 & 3 & 0 & 14 & 1 & 3 & 3 & 5 & 0 & 3 & 3 \\
\hline
\end{tabular}

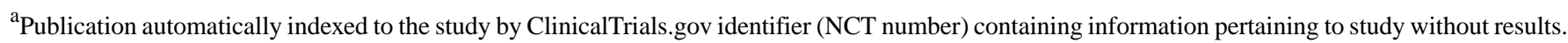
${ }^{\mathrm{b}}$ Publication automatically indexed to the study by ClinicalTrials.gov identifier (NCT number) containing information pertaining to study with results. ${ }^{\mathrm{c}} \mathrm{N} / \mathrm{A}$ : not applicable. 
Table 2. Altered outcomes.

\begin{tabular}{ll}
\hline Disorder and study title & Altered outcomes \\
\hline Stroke &
\end{tabular}

Effects of Patient-centered Stroke Educating System: A Randomized Controlled Trial [152]

Empowerment and Mobile Technology in the Control of Cardiovascular Risk Factors in Patients with Ischemic Stroke (CARDIOSTROKE) [24]

\section{Migraine}

RELAXaHEAD for Headache Patients [33]

\section{Major depressive disorder}

mHealth for Antenatal Mental Health [112]

Text-Message-Based Depression for High-Risk Youth in the ED [41]

Behavioural Activation-Based Treatment Administered Through Smartphone [114]

Mobile Technology to Engage and Link Patients and Providers in Antidepressant Treatment (MedLink) [116]
- Original Primary Outcome Measures (submitted: October 28, 2015): Stroke Health Education Knowledge (time frame: 2 weeks) Changed August 16, 2018 : Stroke Health Education Knowledge Questionnaire (time frame: 4 weeks)

- Original Primary Outcome Measures (submitted: October 17, 2018) was Atrial Fibrillation (3 weeks) as detected in a 3 -week ECG ${ }^{\mathrm{b}}$ monitoring period and Systolic/diastolic blood pressure (time frame: 12 months) measured as difference in systolic/diastolic blood pressure.

- Current Primary Outcome Measures (submitted: October 18, 2018) are Number of Participants with New Atrial Fibrillation and Change in Blood Pressure.

- Current Secondary Outcome Measures (submitted: October 18, 2018)-Number of participants was added before new cardiovascular events within 12 months and new cardiovascular events within 36 months.

- Changed December 29, 2017: Proportion of patients who enrolled in the study/were recruited for the study was eliminated as a primary outcome measure. Satisfaction using Likert scale questions on RELAXaHEAD usability, content, functionality was added as a primary outcome measure.

- $\quad$ Submitted September 9, 2016: Adherence to sampling protocol (time frame: assessed after 6 months) was added as a primary outcome measure.

- Submitted: January 5, 2015: $\Delta$ in Depressive Symptoms was the primary outcome measure. Submitted November 7, 2017- $\Delta$ in Peer Violence Involvement was added as a primary outcome measures.

- $\quad$ Submitted January 5, 2015-original secondary outcome measures was $\Delta$ in Peer Violence Involvement.

- Submitted January 5, 2015: Acceptability/Feasibility: Follow Up Rate, Acceptability/Feasibility: Engagement of Intervention Group and Acceptability/Feasibility: Participant Questionnaire were the secondary outcome measures.

- Original Primary Outcome Measures (submitted: October 31, 2011) was the Montgomery Asberg Depression Rating Scale-Self Rated (MADRS). Current Primary Outcome Measures (submitted: March 22, 2013) is the PHQ- $9^{c}$ and Beck Depression Inventory (BDI).

- Original Secondary Outcome Measures (submitted: March 22, 2013) was the $\mathrm{QOLI}^{\mathrm{d}}, \mathrm{AAQ}^{\mathrm{e}}, \mathrm{BAI}^{\mathrm{f}}$ and TIC-P ${ }^{\mathrm{g}}$. Current Secondary Outcome Measures (submitted March 22, 2013) eliminated the AAQ and $\mathrm{BDI}^{\mathrm{h}}$ as secondary outcome measures.

- Original Primary Outcome Measures (submitted: October 20, 2015) was Adherence to Antidepressant Medication measured as the number of days medication was taken when a dose was expected. Current Primary Outcome Measures (submitted: February 14, 2018) is Adherence to Antidepressant Medication measured through $\%$ of days adherent on Wisepill pillbox as well as self-reported adherence.

- Original Secondary Outcome Measures (submitted: October 20, 2015) was changes in depression measured through self-report PHQ-9 and usability measured through Likert scale ratings. Current secondary outcome measures (submitted: February 14, 2018) is the PHQ-9 and Quick Inventory of Depressive Symptomology Clinician Rating (QIDS-C). 
Disorder and study title ${ }^{\mathrm{a}}$

Mobile Technology to Engage and Link Patients and

Providers in Antidepressant Treatment [117]

Lifestyle Intervention for Young Adults with Serious Mental Illness [57]

Treating Depression on a Day-to-day Basis: Developmen of a Tool for Physicians Based on a Smartphone Application [122]

Using Mental Health Telemetry to Predict Relapse and Rehospitalization in Mood Disorders (PATH-MOD) [54]

IntelliCare: Artificial Intelligence in a Mobile Intervention for Depression and Anxiety (AIM) [125]

Enhancing Delivery of Problem Solving Therapy Using SmartPhone Technology [128]

Technology Assisted Programs that Promote Mental Health for Teenagers (ProjectTECH) [48]

Study of Technology-assisted Treatment of Adolescent Depression (iTAD) [49]

Mobile Phone Sensing and Outreach as Adjuncts to Internet Based Behavior Intervention for Depression [129]

Effectiveness of a Technology Assisted Behavioral Intervention in Assisting People with Major Depressive Disorder [50]
Altered outcomes

- Original Primary Outcome Measures (submitted: July 24, 2013) was Adherence to Medication as measured by when the provided pill bottle is opened to remove a dose of medication and $\Delta$ in Depression Over Time through the PHQ-9. Current Primary Outcome Measures (submitted: January 12, 2015) is measured as Adherence to Antidepressant Medication measured as the frequency of medication usage from baseline to end of treatment.

- Original Secondary Outcome Measures (submitted: July 24, 2013) was Presence of Side Effects and $\Delta \mathrm{s}$ Over Time measured by the Patient Rated Inventory of Side Effects (PRISE) and Frequency, Intensity and Burden of Side Effects Rating (FIBSER). Current Secondary Outcome Measures (submitted: January, 12 2015) is changes in depression measured as the severity of depressive symptoms from baseline to end of treatment.

- $\quad$ Submitted: June 27, 2016

- $\Delta$ in serum lipids was added as a current secondary outcome measures.

- Original Primary Outcome Measures (submitted: September 18, 2018) was comparison between the two groups of the number of participants with a decrease in HDRSS $^{\mathrm{i}}$ scores of at least $50 \%$ at 8 weeks. Current Primary Outcome Measures (submitted: September 20,2018) is a greater clinical response in the active group (smartphone application) comparatively to the comparator group (clinical response was defined as a decline in HDRS- $17^{\mathrm{j}}$ score greater than $50 \%$ ).

- Submitted: November 21, 2014

- Quality-of-Life in Bipolar Disorder was added as a Primary Outcome Measures.

Original primary outcome measures (submitted: June 25, 2014) were changes in depression severity, adherence to mobile application intervention and changes in anxiety. Current Primary Outcome Measures (submitted: February 14, 2018) are the PHQ-9 and GAD- ${ }^{\mathrm{k}}$. Original Secondary Outcome Measures was participant satisfaction. Current Secondary Outcome Measures is the Mean Number of Treatment App Use Sessions by Study Week.

- Original Primary Outcome Measures (submitted: June 28, 2013) was Depression, Anxiety and Stress, an instrument that measures clinical indices of depression and anxiety as well as acute stress. Current Primary Outcome Measures (submitted: July 27, 2016) is the Depression Anxiety and Stress Scale (DASS).

Original Primary Outcome Measures (submitted: July 30, 2013) were Depression (Time Frame: Throughout participation, lasting up to 26 weeks) as measured by the CES-D ${ }^{1}$ and MINIKid and Usability of the Program as measured by the Usefulness, Satisfaction and Ease of Use Questionnaire survey

- Original Secondary Outcome Measures (submitted: April 19,2012) was preliminary indicators of program efficacy (Time Frame: 12 weeks) measured by the 1. Depression Knowledge Test 2. Skill Self Efficacy Questionnaire 3. The Therapeutic Alliance Scale for Adolescents 4. The Cognitive Therapy Scale 5. Acceptance Questionnaire

- Original Primary Outcome Measures (submitted: April 19, 2010) was Depression, as assessed by Quick Inventory of Depressive Symptoms, PHQ-9 and the Mini International Neuropsychiatric Interview Major Depressive Disorders Module (Time Frame: Measured as baseline, 4 and 8 weeks)

- Original Secondary Outcome Measures (submitted: April 19, 2010) was Utilization-Adherence markers for the mobile phone (eg-number of responses to prompts for information) the website (Time frame: Measured from baseline to 8 weeks), Positive Affect, Anxiety (GAD-7) and Health-Related Quality of Life. 
Online Peer Networked Collaborative Learning for Managing Depressive Symptoms (MoodTech) [51]

\section{Anxiety}

Connection to Care: Pilot Study of a Mobile Health Tool for Patients with Depression and Anxiety [131]

Improving Medical Care With Electronic Interventions Based on Automated Text and Phone Messages [132]

Effect of Premedication Type on Preoperative Anxiety in Children [77]

Using Smartphones to Enhance the Treatment of Childhood Anxiety [80]

Youth Mayo Clinic Anxiety Coach Pilot Study [81]

ACT-smart: Smartphone-supplemented iCBT for Social Phobia and/or Panic Disorder [82]
- Original Primary Outcome Measures (submitted: July 18, 2008) was Depression, as assessed by HRSD ${ }^{\mathrm{m}}$, PHQ-9 and SCID and Attrition (in arms with i-CBT ${ }^{\mathrm{n}}$ )

- Original Secondary Outcome Measures was i-CBT utilization (eg, number of logins, average visit length, total time spent on the site, number of exercises completed), Health-Related Quality of Life $\left(\mathrm{SF}-36 \mathrm{~V}^{\mathrm{O}}\right)$ and Patient Satisfaction (Satisfaction Index-Mental Health)

- Original Primary Outcome Measures (submitted: July 20, 2016) was Depression (Time Frame: Baseline to end of treatment) measured as the $\Delta$ in self-reported depressive symptom severity from baseline to end of treatment and adherence to the program measured as the number of times the program is accessed from start to last use from baseline to end of treatment

Original Primary Outcome Measures (submitted: July 13, 2015) were patient acceptability as determined by qualitative interview, care manager acceptability as determined by qualitative interview, patient report of usefulness as determined by qualitative interview and care manager report of usefulness as determined by qualitative interview. Current primary outcome measures (submitted: October 25,2017 ) are app acceptability as measured by number of patient app users who rate app easy to use and time spent reasonable, app acceptability as measured by number of care manager dashboard users who rate dashboard easy to use and time spent reasonable, app usefulness as measured by number of patient app users who rate app easy to use and time spent reasonable and app usefulness as measured by number of care manager dashboard users who rate dashboard as useful.

- Current Secondary Outcome Measures (submitted: February 10, 2017)-Breastfeeding duration was added as a current secondary outcome measure.

- Current Primary Outcome Measures (submitted: August 13, 2018)-Mask acceptance (At anesthesia induction) was eliminated as a primary outcome measure and added as a secondary outcome measure.

Original Primary Outcome Measures (submitted: October 3, 2014) was the Clinical Global Impression Improvement (CGI-I) defined as treatment response at post treatment. Current Primary Outcome Measures (submitted: January 5, 2016) is the PARS ${ }^{\mathrm{p}}$ Treatment response. Original Secondary Outcome Measures was the PARS and Screen for Childhood Anxiety Related Emotional Disorders (SCARED). Current Secondary Outcome Measures is the Absence of diagnosis on $\mathrm{K}_{-S A D S}^{\mathrm{q}}$

- Current Primary Outcome Measures (submitted: July 30, 2014)-qualitative interview assessing subject safety and treatment adherence (Time frame: within 5 working days of treatment completion) was eliminated as a primary outcome measure

- Current Primary Outcome Measures and Current Secondary Outcome Measures (submitted: November 27, 2013)

- All outcomes that listed month 24 after the treatment period in the time frame were changed to month 36 after the treatment period. 


\begin{tabular}{|c|c|}
\hline Disorder and study title ${ }^{\mathrm{a}}$ & Altered outcomes \\
\hline $\begin{array}{l}\text { mWELLCARE: An Integrated mHealth System for the } \\
\text { Prevention and Care of Chronic Disease (mWELLCARE } \\
\text { [134] }\end{array}$ & $\begin{array}{l}\text { - Changed September 12, 2016: no longer tracking 10-year risk of } \mathrm{CHD}^{\mathrm{r}} \text { and } \\
\text { added tracking for alcohol use, fasting blood sugar, total cholesterol, } \mathrm{CVD}^{\mathrm{S}} \text { risk, } \\
\text { and cost }\end{array}$ \\
\hline $\begin{array}{l}\text { Project Guard: Reducing Alcohol Misuse/Abuse in the } \\
\text { National Guard [88] }\end{array}$ & - Updated August 8, 2016: No changes \\
\hline $\begin{array}{l}\text { Skills-Training for Reducing Risky Alcohol Use in App } \\
\text { Form [137] }\end{array}$ & $\begin{array}{l}\text { - Updated October 23, 2018: no longer looking for reduction in alcohol consump- } \\
\text { tion }\end{array}$ \\
\hline $\begin{array}{l}\text { Usefulness of Supportive Text Messages in the Treatment } \\
\text { of Depressed Alcoholics [96] }\end{array}$ & $\begin{array}{l}\text { - Updated December 17, 2011: Becks Depression inventory Score was added, } \\
\text { and global assessment of function score was added }\end{array}$ \\
\hline \multicolumn{2}{|l|}{ Epilepsy } \\
\hline Embrace: Seizure Characterization [39] & - Updated June 22, 2018: Original measures not given \\
\hline \multicolumn{2}{|l|}{ Schizophrenia } \\
\hline $\begin{array}{l}\text { Development of a Mobile System for Self-Management of } \\
\text { Schizophrenia (SOS) [60] }\end{array}$ & - $\quad$ Updated June 2, 2016: No longer tracking medication adherence. \\
\hline $\begin{array}{l}\text { A New Paradigm for Illness Monitoring and Relapse Pre- } \\
\text { vention in Schizophrenia [63] }\end{array}$ & $\begin{array}{l}\text { - Updated June 2, 2016: Now using BRPS to assess psychotic symptom severity } \\
\text { instead of PANSS }\end{array}$ \\
\hline \multicolumn{2}{|l|}{ Other mental and substance use disorders } \\
\hline $\begin{array}{l}\text { Preventing HIV/STI in Urban Adolescents via an mHealth } \\
\text { Primary Care Intervention [148] }\end{array}$ & - Updated February 12, 2018: No longer tracking $\Delta$ in adolescent $\mathrm{STI}^{\mathrm{u}}$ testing. \\
\hline $\begin{array}{l}\text { CopeSmart: Using Mobile Technology to Promote Positive } \\
\text { Mental Health In Young People [149] }\end{array}$ & - Updated October 10, 2014: Changed to use Emotional Self-Awareness Scale. \\
\hline
\end{tabular}

${ }^{\text {a }}$ Publication automatically indexed to the study by ClinicalTrials.gov identifier (NCT number) without results.

${ }^{\mathrm{b}} \mathrm{ECG}$ : electrocardiogram.

${ }^{\mathrm{c}}$ PHQ-9: Patient Health Questionnaire-9.

dQOLI: Quality of Life Inventory.

${ }^{\mathrm{e}} \mathrm{AAQ}$ : Swiss Agency of Accreditation and quality assurance.

${ }^{f}$ BAI: Beck Anxiety Inventory.

${ }^{g}$ TIC-P: Trimbos and iMTA questionnaire on Costs associated with Psychiatric illness.

hDI: Beck Depression Inventory.

${ }^{\mathrm{i}}$ HDRSS: HDRS Hamilton Depressive Rating Scale.

${ }^{\mathrm{j}}$ HDRS-17: HDRS Hamilton Depressive Rating Scale.

${ }^{\mathrm{k}}$ GAD-7: General Anxiety Disorder-7.

${ }^{1}$ CES-D: Center for Epidemiological Studies-Depression.

${ }^{m}$ HRSD: Hamilton Rating Scale for Depression.

$\mathrm{n}_{\mathrm{i}-\mathrm{CBT}}$ : internet-based Cognitive Behavioral Therapy.

${ }^{\circ}$ SF-36V: Satisfaction Index-Mental Health.

PARS: Pediatric Anxiety Rating Scale.

${ }^{\mathrm{q}}$ K-SADS: DSM 5 Diagnosis of Separation Anxiety, Social Anxiety, and Generalized Anxiety Disorder on the K-SADS interview.

${ }^{\mathrm{r}} \mathrm{CHD}$ : coronary heart disease.

${ }^{\mathrm{s}} \mathrm{CVD}$ : cardiovascular disease.

tPANSS: Positive and Negative Syndrome Scale.

${ }^{\mathrm{u}} \mathrm{STI}$ : sexually transmitted infection.

The second table shows that the studies were first registered in 2008 , with more than half of the studies registered between 2016 and 2018. Across all 135 studies, the mean estimated enrollment was 1078 , although the median was only 100 . Across all 135 studies, the actual reported enrollment was lower, with a mean of 249 and median of 80 . Only about a quarter (35/135, $25.9 \%$ ) of the 135 studies were NIH funded.
Of the 135 studies included, only $9(6.7 \%)$ studies reported their results. A total of $16.9 \%$ (23/135) of studies had publications automatically indexed to the ClinicalTrials.gov identifier (Multimedia Appendix 1). Moreover, 18.5\% (25/135) of trials had results reported in some publicly accessible location (either the results section of ClinicalTrials.gov or in publications 
indexed in databases, which were automatically associated with the ClinicalTrials.gov identifier; Multimedia Appendix 1).

Of those studies that were registered more than 3 years ago (a time frame the authors deemed reasonable given (1) the generally short study interventions, (2) the rapidly changing mHealth landscape, and (3) the 1-year time frame that ClinicalTrials.gov gives to post results after study completion), $14.5 \%(8 / 55)$ of the studies had results posted on ClinicalTrials.gov. The conditions and the number of studies for which the results were published are stroke (1/11), major depressive disorder (4/15), anxiety disorders (2/7), and schizophrenia (1/6). Of the 135 studies, 33 (24.4\%) studies that altered their outcomes after the original outcome measures were posted (Multimedia Appendix 2). Of the 135 studies, there were $45(33.3 \%)$ studies that were marked completed by October 2017,1 year before the search date. Of the 45 studies, 20 (44\%) had results reported in some publicly accessible location (either the results section of ClinicalTrials.gov or in publications indexed in databases, which were automatically associated with the ClinicalTrials.gov identifier). Moreover, $15 \%$ (7/45) of studies reported their results on ClinicalTrials.gov, and $30 \%$ (14/45) of studies had publications automatically indexed to the ClinicalTrials.gov identifier.

There was a statistically significant relationship, as determined by a two-sided $t$ test, with studies reporting results having a shorter mean duration (121.7 days) compared with studies never reporting results (153.8 days; $P<.001)$. There was also a statistically significant relationship with studies reporting results having lower actual enrollment (142.3 people) compared with studies never reporting results (295.6 people; $P=.01$ ). There was no statistically significant relationship between the estimated enrollment at the time of study registration and never reporting results. There was also no statistically significant relationship between the status of a study and its estimated or actual enrollment or length of intervention.

\section{Discussion}

\section{Principal Findings}

In this comprehensive analysis of registered mHealth studies of interventions for disabling neuropsychiatric conditions, there were 6 key findings. First, there has been a large increase in the number of clinical trial registrations in the past 2 years; almost half $(44.5 \%)$ of the trial registrations were registered in the past 2 years. Thus, despite the increasing additions of health-based smartphone apps [1], this snapshot of ClinicalTrials.gov suggests that only a few such apps for high-burden neuropsychiatric conditions are being clinically evaluated in trials. Second, the studies were generally located in the United States, but a few of the studies were funded by the NIH. Third, the study characteristics were such that they would not generally be considered as high-quality evidence and for use in guideline recommendations because of small sample sizes and heterogeneous interventions. Fourth, the mean study duration has not changed with time, suggesting that long-term outcomes are still not the focus of research. Despite the myriad of ways in which results can be reported, a few trials had results reported either as entered on ClinicalTrials.gov or as study results automatically indexed to ClinicalTrials.gov. As stated earlier, only $6.7 \%(9 / 135)$ of such trials that have been registered on ClinicalTrials.gov have reported results. Overall, $18.5 \%$ (25/135) of trials had results reported in some capacity (either under the results section of ClinicalTrials.gov or through publications indexed in databases, which were automatically associated with the ClinicalTrials.gov identifier). Fifth, initially specified outcomes were changed after trials commenced in a quarter of all trials. Finally, study duration and sample size of those enrolled are associated with the reporting results of studies.

More trials are being registered on ClinicalTrials.gov, given the requirements for prospective registration as a condition of publication. A previous study examined clinical trial registration for 3 groups of disorders (ie, cardiovascular disorders, mental health disorder, and oncologic disorders) that comprise the largest number of DALYs lost in the United States. The authors found that the number of trials submitted for registration between October 2004 and September 2007 and then October 2007 and September 2010 increased by about $140 \%$ from 28,881 to 40,970 [9]. A study assessing trial registration revealed that a few trials still reported on the trial registries [154]. However, more researchers are learning about this requirement, hence the expected increase in trial registrations.

Despite the increase in trial registrations, only 135 trials met the criteria for this study. Thus, a few apps for high-burden neuropsychiatric conditions are being clinically evaluated in the trials reported on ClinicalTrials.gov. A recent study of top-funded digital health companies examined the number of research studies collectively undertaken and found that of the top 10 disease categories examined, 7 were neuropsychiatric. However, none of these industry studies reported on the clinical effectiveness of the digital health tools for these high-burden conditions. Thus, although there is high interest in digital health toward neuropsychiatric conditions, there is little registered evidence that such apps on the market work [155].

A majority of the studies were based in the United States, followed by Europe. This is not because ClinicalTrials.gov is based in the United States, as ClinicalTrials.gov accounts for more than $80 \%$ of all the clinical studies in the WHO portal [9]. There may be fewer studies in Europe because of the newly implemented General Data Protection Regulation in 2018, which is a European Union (EU) law for the protection of data and privacy for all individuals within the EU and the European Economic Area. It also concerns the export of personal data outside the EU.

A few $(26 \%)$ of the trials were NIH funded. This is not surprising as much of the research in digital health has occurred in the private sector. In 2016, 296 private digital health companies received venture funding that totaled to more than US \$4.2 billion and approached US \$6 billion in 2017 [155]. The 20 top-funded, private US-based digital health companies were studied to analyze their products and services, peer-reviewed evidence, and the potential for impact on patients with high-burden conditions. Less than one-third (27.9\%) of the studies targeted patients with a high-burden condition. Only $16(15 \%)$ studies assessed the clinical effectiveness of the product or service, and only $8(8 \%)$ studies assessed the clinical 
effectiveness of the product or service in a high-burden or high-risk factor population. Only a small number of studies published data, and interestingly, journals without impact factors were the most common (31\%) source of publications [155].

There was tremendous heterogeneity in the purpose of the use of the apps not only across conditions but also within a given condition. The most common purpose across all conditions was symptom tracking and medication adherence. There were also some studies based on skills learning, for example, cognitive behavioral therapy or progressive muscle relaxation, and an app was used to help with the delivery of these skills. A small number of studies were designed to detect physical symptoms or signs, such as atrial fibrillation and seizure, or enhance communication with clinicians using apps connected to sensors and/or devices. This latter intervention was to provide support to patients, in some cases with the intent to prevent relapse or indicate the degree of symptoms to potentially lead to a change in medication management if warranted.

In terms of study design, the studies were generally small (samples sizes<100). This study's results were comparable with the study examining the ClinicalTrials.gov registration of interventional studies for cardiovascular disease, mental health, and oncology, which found that $62 \%$ of the trials had an anticipated enrollment mean of less than 100 [9]. This study's findings were also comparable with a study of trials conducted by the top 20 funded digital health companies, which found that $51 \%$ of the studies had less than 100 participants [155]. Thus, these studies will not be able to help in creating major guidelines with high-quality evidence related to these disabling neuropsychiatric conditions.

Although this study was not designed to assess why there is low registration of trials, the potential reasons may include lack of awareness and different prioritization in the app development industry. "Given the diversity of stakeholders involved in mHealth research, competing outcomes, priorities, funding, and publication requirements may potentially mean some studies are less likely to be registered" [156].

This study's results are in line with previous work showing that few studies reported published results on ClinicalTrials.gov. In a study examining trial registration compliance in publications related to headache, only $26 \%$ of all the studies that should have been registered were indeed registered [154]. A recent study of 556 trial registrations on ClinicalTrials.gov showed that out of all the trials in the study, $150(27 \%)$ trials remained unpublished 5 years after the study completion dates [157]. There are a number of potential reasons for the low publication rates. First, the lack of reporting of negative results is a well-known phenomenon in academic medicine $[6,158,159]$. For example, some trials of triptans [160] and gabapentin [154,161] were never published. Second, in mHealth, there are likely specific challenges to publication, including high attrition rates, usability issues, and lack of sufficient previous formative research [162]. Third, previous research has shown that clinical trials with large sample sizes were more likely to be published $[154,158,162,163]$. Many of the mHealth clinical trial registrations in this study's sample sizes were considerably small.
As noted previously, the study outcomes were changed. In a systematic review in 2011 assessing the transparency of outcome reporting and trial registration of randomized controlled trials and top psychosomatic and behavioral health journals, of the 63 articles meeting the study criteria, only $25(39.7 \%)$ articles had adequately declared primary or secondary outcomes [164]. Thus, this study's results are in line with previous research in the field of mental health. This is an especially prevalent concern in digital health, where researchers could easily change their outcomes and conduct selective analyses [165].

Finally, this study's findings suggest that study status is not associated with either estimated or actual sample size or length of the intervention. Study status may not be updated in real time; thus, it may not reflect the true status of the study. This study's findings that studies never reporting any results have a longer duration than those reporting results are logical in the sense that shorter studies may be easier to complete. Similarly, this study's findings that studies never reporting any results have a higher mean number of participants compared with studies that did report results make sense, as larger studies are more likely to be difficult to complete. In sum, these findings that shorter and smaller studies are associated with reporting results compared with longer and larger studies are intuitive and reflect that digital health studies have the same challenges that all clinical studies face in terms of reaching reporting status.

\section{Limitations}

One of the limitations of this study is that we may have failed to identify some mHealth studies due to the sampling methodology. The primary search terms that we used were "mHealth," "smartphone," "electronic diary," and "mobile technology," while specifying subcategories of the different neuropsychiatric conditions (ie, migraine, migraine with aura, migraine without aura, and migraine disorders). Other search terms such as "digital," "ecological momentary assessment," "experience sampling," or "log" may have captured additional studies. We only searched ClinicalTrials.gov, and other trial registration websites exist. Second, many of the studies are recent, and thus authors may not have completed their studies and posted results yet. Third, the potential usefulness of different behavioral interventions administered through smartphone apps is not listed on ClinicalTrials.gov and is likely unknown; it is generally the point of the studies to assess whether these interventions may be effective. Finally, the information on ClinicalTrials.gov will always be incomplete for two main reasons: (1) Individual studies may not be registered in the database. Second, information entered on ClinicalTrials.gov may be incomplete, for example, certain data elements may have had a different format or structure or may have been optional when the study information was initially entered. (2) There are few incentives to motivate responsible parties to update their studies [150] registered on ClinicalTrials.gov. One study found that $17 \%$ to $20 \%$ of the studies on ClinicalTrials.gov were observational and only $7 \%$ had posted results $[150,151]$. Inferential statistics were limited based on reports from individual studies. 


\section{Future Directions}

The study information on ClinicalTrials.gov is helpful for understanding the landscape of smartphone-based studies for neuropsychiatric conditions. However, the information listed does not offer enough detail to fully understand the nature of smartphone apps and sensor data collection. Future solutions may include the posting of web-based demonstrations of apps being studied or links to the version of the app used in trials. In addition, it is clear that many of the health-based apps are being developed by the private sector. Efforts need to be made to encourage commercial companies to register their studies on ClinicalTrials.gov and to adhere to the trial registration guidelines, for example, report trial results in a timely manner, as described by ClinicalTrials.gov. For health-based apps that make claims of efficacy, there needs to be stringent oversight of the registered clinical trials.

\section{Conclusions}

Despite the increasing use of health-based smartphone apps by the general public, only a few such apps are rigorously evaluated in clinical settings. Similar to other research on the studies registered on ClinicalTrials.gov, studies of the top neuropsychiatric conditions involving mHealth, registered on ClinicalTrials.gov, tended to be small, and there was a large amount of heterogeneity in the methods (types of interventions), duration, and reporting methods [9]. Moreover, very few registered studies $(6.7 \%)$ reported their results, raising the question of whether the burgeoning creation of mHealth-based interventions is efficacious, despite these apps being widely downloaded and used. There were few studies for the most disabling neuropsychiatric conditions that typically use electronic diaries for the self-management of migraine and epilepsy. Future work should focus on studying the efficacy of these mHealth interventions for neuropsychiatric conditions if they are to be used by patients with these disabling conditions. Such studies should be registered on ClinicalTrials.gov to ensure transparency and so that the public can also learn about the research being conducted using these interventions.

\section{Acknowledgments}

Salary support for MM was funded by a grant from NIH NCCIH K23 AT009706.

\section{Authors' Contributions}

Study conception was done by MM, EL, and JT. JR and PP conducted data analysis and performed descriptive analyses. Statistical analysis was conducted by JT, JR, and PP. Manuscript drafting was done by MM, JR, and PP, with all authors MM, JR, PP, EL, and $\mathrm{JT}$ revising it for intellectual content.

\section{Conflicts of Interest}

Both MM and JT have NIH-funded studies to conduct app-based research.

\section{Multimedia Appendix 1}

Study methodology, key findings, and data collected.

[DOC File, 837 KB-Multimedia Appendix 1]

\section{Multimedia Appendix 2}

Mobile health studies, study criteria, and altered outcomes.

[DOC File, 237 KB-Multimedia Appendix 2]

\section{References}

1. Pohl M. 325,000 Mobile Health Apps Available in 2017 - Android Now the Leading mHealth Platform. Research2Guidance. 2017. URL: https://research2guidance.com/325000-mobile-health-apps-available-in-2017/ [accessed 2020-06-17]

2. mHealth Apps Market Size Worth \$236.0 Billion By 2026. Grand View Research. 2019. URL: https://www. grandviewresearch.com/press-release/global-mhealth-app-market [accessed 2020-06-17]

3. Bakker D, Kazantzis N, Rickwood D, Rickard N. Mental health smartphone apps: review and evidence-based recommendations for future developments. JMIR Ment Health 2016 Mar 1;3(1):e7 [FREE Full text] [doi: 10.2196/mental.4984] [Medline: 26932350]

4. Schwartz JL. Real-world evidence, public participation, and the FDA. Hastings Cent Rep 2017 Nov;47(6):7-8. [doi: 10.1002/hast.779] [Medline: 29171057]

5. Larsen ME, Huckvale K, Nicholas J, Torous J, Birrell L, Li E, et al. Using science to sell apps: evaluation of mental health app store quality claims. NPJ Digit Med 2019;2:18 [FREE Full text] [doi: 10.1038/s41746-019-0093-1] [Medline: 31304366]

6. Brassington I. The ethics of reporting all the results of clinical trials. Br Med Bull 2017 Jan 1;121(1):19-29. [doi: 10.1093/bmb/ldw058] [Medline: 28104631]

7. Registration of Clinical Trials on ClinicalTrials.gov. Johns Hopkins Medicine. 2019. URL: https://www.hopkinsmedicine.org/ institutional_review board/guidelines policies/guidelines/clinical trials.html [accessed 2020-06-17] 
8. FDAAA 801 and The Final Rule. Clinical Trials. 2018. URL: https://clinicaltrials.gov/ct2/manage-recs/ fdaaa\#WhichTrialsMustHaveResults [accessed 2018-12-31]

9. Califf RM, Zarin DA, Kramer JM, Sherman RE, Aberle LH, Tasneem A. Characteristics of clinical trials registered in ClinicalTrials.gov, 2007-2010. J Am Med Assoc 2012 May 2;307(17):1838-1847. [doi: 10.1001/jama.2012.3424] [Medline: 22550198]

10. Ross JS, Tse T, Zarin DA, Xu H, Zhou L, Krumholz HM. Publication of NIH funded trials registered in ClinicalTrials.gov: cross sectional analysis. Br Med J 2012 Jan 3;344:d7292 [FREE Full text] [doi: 10.1136/bmj.d7292] [Medline: 22214755]

11. GBD Results Tool. Global Health Data Exchange. 2019. URL: http://ghdx.healthdata.org/gbd-results-tool [accessed 2019-11-16]

12. Whiteford HA, Degenhardt L, Rehm J, Baxter AJ, Ferrari AJ, Erskine HE, et al. Global burden of diasease attributable to mental and substance use disorders: findings from the global burden of disease study 2010. Lancet 2013 Nov 9;382(9904):1575-1586. [doi: 10.1016/S0140-6736(13)61611-6] [Medline: 23993280]

13. Murray C, Vos T, Lozano R, Naghavi M, Flaxman AD, Michaud C, et al. Disability-adjusted life years (DALYs) for 291 diseases and injuries in 21 regions, 1990-2010: a systematic analysis for the global burden of disease study 2010. Lancet 2012 Dec 15;380(9859):2197-2223. [doi: 10.1016/S0140-6736(12)61689-4] [Medline: 23245608]

14. Minen M, Azarchi S, Sobolev R, Shallcross A, Halpern A, Berk T, et al. Factors related to migraine patients' decisions to initiate behavioral migraine treatment following a headache specialist's recommendation: a prospective observational study. Pain Med 2018 Nov 1;19(11):2274-2282 [FREE Full text] [doi: 10.1093/pm/pny028] [Medline: 29878178]

15. Fairburn CG, Patel V. The impact of digital technology on psychological treatments and their dissemination. Behav Res Ther 2017 Jan;88:19-25 [FREE Full text] [doi: 10.1016/j.brat.2016.08.012] [Medline: 28110672]

16. Chandrashekar P. Do mental health mobile apps work: evidence and recommendations for designing high-efficacy mental health mobile apps. Mhealth 2018;4:6 [FREE Full text] [doi: 10.21037/mhealth.2018.03.02] [Medline: 29682510]

17. Impact of M-health based Intervention on Adherence to Healthy Physical Activity After Stroke. Clinical Trials. 2018. URL: https://ClinicalTrials.gov/ct2/show/

NCT03507894?cond=Impact+of+M-health+based+Intervention+on+Adherence+to+Healthy+Physical+Activity+After+Stroke\&rank=1 [accessed 2018-11-27]

18. Increasing Physical Activity in Stroke Survivors Using STARFISH. Clinical Trials. 2017. URL: https://ClinicalTrials.gov/ ct2/show/NCT02494245? cond=Increasing+Physical+Activity+in+Stroke+Survivors+Using+STARFISH\&rank=2 [accessed 2018-11-27]

19. The Stroke and Exercise Program. Clinical Trials. 2017. URL: https://ClinicalTrials.gov/ct2/show/ NCT02701998?cond=The+Stroke+and+Exercise+Program\&rank=1 [accessed 2018-11-27]

20. Stroke Inpatient Rehabilitation Reinforcement of ACTivity. Clinical Trials. 2010. URL: https://ClinicalTrials.gov/ct2/show/ NCT01246882?cond=Stroke+Inpatient+Rehabilitation+Reinforcement+of+ACTivity\&rank=1 [accessed 2018-11-27]

21. Survey of Lifelong Food and Nutrition Assistance (LIFANA) in Stroke Patients and Caregivers. Clinical Trials. 2018. URL: https://ClinicalTrials.gov/ct2/show/NCT03635476? cond=LIFANA\&rank=1 [accessed 2018-11-27]

22. mHealth Screening to Prevent Strokes (mSToPS). Clinical Trials. 2015. URL: https://ClinicalTrials.gov/ct2/show/ NCT02506244? cond=mstops\&rank=1 [accessed 2018-11-27]

23. VR-3D Movie-Based Education. Clinical Trials. 2017. URL: https://ClinicalTrials.gov/ct2/show/ NCT03104231? cond=vr+3d\&rank=1 [accessed 2018-11-27]

24. Empowerment and Mobile Technology in the Control of Cardiovascular Risk Factors in Patients With Ischemic Stroke (CARDIOSTROKE). Clinical Trials. 2018. URL: https://ClinicalTrials.gov/ct2/show/ NCT03710902?term=NCT03710902\&rank=1 [accessed 2018-11-27]

25. Hispanic Secondary Stroke Prevention Initiative (HISSPI). Clinical Trials. 2014. URL: https://ClinicalTrials.gov/ct2/show/ NCT02251834?cond=hispanic+stroke\&rank=1 [accessed 2018-11-27]

26. Phone-Based Intervention Under Nurse Guidance After Stroke (PINGS). Clinical Trials. 2015. URL: https://ClinicalTrials. gov/ct2/show/NCT02568137? cond=Phone-based+Intervention+Under+Nurse+Guidance+After+Stroke\&rank=1 [accessed 2018-11-27]

27. Technology Intervention to Support Caregiving for Alzheimer's Disease (I-CARE) (I-CARE). Clinical Trials. 2017. URL: https://ClinicalTrials.gov/ct2/show/

NCT03119259? cond=iCare-AD\%3A+A+Mobile+Health+Application+for+Caregivers+of+Patients+With+Dementia\&rank=1 [accessed 2018-11-27]

28. Diabetes as an Accelerator of Cognitive Impairment and Alzheimer's Disease (DIALCAT). Clinical Trials. 2018. URL: https://ClinicalTrials.gov/ct2/show/NCT03578991 [accessed 2018-11-27]

29. Comparing Smartphone Technology and a Memory Strategy on Improving Prospective Memory in Alzheimer's Disease. Clinical Trials. 2017. URL: https://ClinicalTrials.gov/ct2/show/

NCT03384043? cond=Comparing+Smarthone+Technology+and+a+Memory+Strategy+on+Improving+Prospective+Memory+in+Altheimer\%27s+Disease\&uank=1 [accessed 2018-11-27]

30. Therapeutic Efficacy of Categorical Language Fluency Smartphone Game Application. Clinical Trials. 2016. URL: https:/ /ClinicalTrials.gov/ct2/show/ 
NCT02848404?cond=Therapeutic+Efficacy + of + Categorical+Language+Fluency + Smartphone + Game+Application\&rank=1 [accessed 2018-11-27]

31. Therapeutic Efficacy of Categorical Language Fluency Smartphone Game Application. Clinical Trials. 2016. URL: https:/ /ClinicalTrials.gov/ct2/show/

NCT02848404?cond=Therapeutic+Efficacy+of+Categorical+Language+Fluency+Smartphone+Game+Application\&rank=1 [accessed 2018-11-27]

32. Developing a Mobile Health Pain-Coping Skills Training Program for the Treatment of Chronic Migraine: AIM 4. Clinical Trials. 2018. URL: https://ClinicalTrials.gov/ct2/show/NCT03465826 [accessed 2018-11-27]

33. RELAXaHEAD for Headache Patients. Clinical Trials. 2017. URL: https://ClinicalTrials.gov/ct2/show/ NCT03183791?cond=RELAXaHEAD+for+Headache+Patients\&rank=1 [accessed 2018-11-27]

34. Improving Health Outcomes of Migraine Patients Who Present to the Emergency Department. Clinical Trials. 2016. URL: https://ClinicalTrials.gov/ct2/show/

NCT02945839?cond=Improving+Health+Outcomes+of+Migraine+Patients+Who+Present+to+the+Emergency+Department\&rank=1 [accessed 2018-11-27]

35. A Study of the Effect of a Disease-Specific Migraine Smart Phone Application (App) on Participant Care. Clinical Trials. 2018. URL: https://ClinicalTrials.gov/ct2/show/NCT03559088 [accessed 2018-11-27]

36. Behavioral and Educational Tools to Improve Epilepsy Care. Clinical Trials. 2016. URL: https://ClinicalTrials.gov/ct2/ show/NCT02646631?cond=Behavioral+and+Educational+Tools+to+Improve+Epilepsy+Care\&rank=1 [accessed 2018-11-27]

37. Stress Management Intervention for Living With Epilepsy (SMILE). Clinical Trials. 2011. URL: https://ClinicalTrials.gov/ ct2/show/NCT01444183?cond=Stress+Management+Intervention+for+Living+with+Epilepsy+\%28SMILE\%29\&rank=1 [accessed 2018-11-27]

38. Generalized Seizure Detection And Alerting In The EMU With The Empatica Embrace Watch And Smartphone-Based Alert System. Clinical Trials. 2017. URL: https://ClinicalTrials.gov/ct2/show/NCT03207685 [accessed 2018-11-27]

39. Embrace: Seizure Characterization. Clinical Trials. 2017. URL: https://ClinicalTrials.gov/ct2/show/NCT03206502?cond=. + Embrace\%3A+Seizure+Characterization\&rank=1 [accessed 2018-11-27]

40. AniMovil mHealth Support for Depression Management in a Low-Income Country (AniMovil). Clinical Trials. 2018. URL: https://ClinicalTrials.gov/ct2/show/

NCT03615118?cond=AniMovil+mHealth+Support+for+Depression+Management+in+Low-Income+Country\&rank=1 [accessed 2018-11-27]

41. Text-Message-Based Depression Prevention for High-Risk Youth in the ED (iDOVE). Clinical Trials. 2015. URL: https:/ /ClinicalTrials.gov/ct2/show/

NCT02332239?cond=Text-Message-Based+Depression+for+High-Risk+Youth+in+the+ED\&rank=1 [accessed 2018-11-27]

42. An Adaptive Intervention for Depression Among Latinos Living With HIV (Latino-SMART). Clinical Trials. 2018. URL: https://ClinicalTrials.gov/ct2/show/

NCT03668379?cond=An+Adaptive+Intervention+for+Depression+Among+Latinos+Living+With+HIV\&rank=1 [accessed 2018-11-27]

43. Intervention to Prevent Peer Violence \& Depressive Symptoms Among At-Risk Adolescents (iDOVE2). Clinical Trials. 2018. URL: https://ClinicalTrials.gov/ct2/show/NCT03626103?term=NCT03626103\&rank=1 [accessed 2018-11-27]

44. Cognitive Behavioral Therapy Treatment of Depression With Smartphone Support. Clinical Trials. 2013. URL: https:/ /ClinicalTrials.gov/ct $2 /$ show/

NCT01819025? cond=Cognitive+Behavioral+Therapy+Treatment+of+Depression+With+Smartphone+Support\&rank=1 [accessed 2018-11-27]

45. Cognitive-Behavioral Intervention Via a Smartphone App for Depressive Symptoms in Caregivers (App Depression). Clinical Trials. 2017. URL: https://ClinicalTrials.gov/ct2/show/

NCT03110991?cond=Cognitive-behavioral+Intervention+via+a+Smartphone+App+for+Depressive+Symptoms+in+Caregivers\&rank=1 [accessed 2018-11-27]

46. Reducing Depressive Symptomatology With a Smartphone App. Clinical Trials. 2017. URL: https://ClinicalTrials.gov/ct2/ show/NCT03060200?term=NCT03060200\&rank=1 [accessed 2018-11-27]

47. Smartphone-Enabled Health Coaching Intervention for Youth Diagnosed With Major Depressive Disorders. Clinical Trials. 2018. URL: https://ClinicalTrials.gov/ct2/show/NCT03406052?term=NCT03406052\&rank=1 [accessed 2018-11-27]

48. Technology Assisted Programs That Promote Mental Health for Teenagers (ProjectTECH). Clinical Trials. 2013. URL: https://ClinicalTrials.gov/ct2/show/NCT01912729?term=NCT01912729\&rank=1 [accessed 2018-11-27]

49. Study of Technology-assisted Treatment of Adolescent Depression (iTAD). Clinical Trials. 2013. URL: https://ClinicalTrials. gov/ct2/show/NCT01582581?term=NCT01582581\&rank=1 [accessed 2018-11-27]

50. Effectiveness of a Technology Assisted Behavioral Intervention in Assisting People With Major Depressive Disorder. Clinical Trials. 2008. URL: https://ClinicalTrials.gov/ct2/show/NCT00719979?term=NCT00719979\&rank=1 [accessed 2018-11-27]

51. Online Peer Networked Collaborative Learning for Managing Depressive Symptoms (MoodTech). Clinical Trials. 2016. URL: https://ClinicalTrials.gov/ct2/show/NCT02841787?term=NCT02841787\&rank=1 [accessed 2018-11-27] 
52. New Technologies for Cognitive Behavior Therapy (CBT) Treatment of Adolescent Depression. Clinical Trials. 2013. URL: https://ClinicalTrials.gov/ct2/show/ NCT01868867?cond=New+Technologies+for+Cognitive+Behavior+Therapy+\%28CBT\%29+Treatment+of+Adolescent+Depression\&rank=1 [accessed 2018-11-27]

53. Mental Health Telemetry for Self-Management in Major Depression (MHTV). Clinical Trials. 2013. URL: https:/ /ClinicalTrials.gov/ct2/show/NCT01999010?term=NCT01999010\&rank=1 [accessed 2018-11-27]

54. Using Mental Health Telemetry to Predict Relapse and Re-hospitalization in Mood Disorders (PATH-MOD). Clinical Trials. 2013. URL: https://ClinicalTrials.gov/ct2/show/NCT01882608?term=NCT01882608\&rank=1 [accessed 2018-11-27]

55. Training and Supervision Program for Depression Management. Clinical Trials. 2014. URL: https://ClinicalTrials.gov/ct $2 /$ show/NCT02232854?cond=Training+and+Supervision+Program+for+Depression+Management\&rank=1 [accessed 2018-11-27]

56. Effectiveness of a mHealth Intervention for the Treatment of Depression in People With Diabetes or Hypertension in Peru (LATIN-MHPeru). Clinical Trials. 2017. URL: https://ClinicalTrials.gov/ct2/show/NCT03026426 [accessed 2018-11-27]

57. Lifestyle Intervention for Young Adults With Serious Mental Illness. Clinical Trials. 2016. URL: https://ClinicalTrials. gov/ct2/show/NCT02815813? cond=Lifestyle+Intervention+for+Young+Adults+with+Serious+Mental+Illness\&rank=1 [accessed 2018-11-27]

58. Comparing Mobile Health (mHealth) and Clinic-Based Self-Management Interventions for Serious Mental Illness. Clinical Trials. 2015. URL: https://ClinicalTrials.gov/ct2/show/NCT02421965?term=NCT02421965\&rank=1 [accessed 2018-11-27]

59. Study of m-RESIST, an m-Health Program for Treatment-Resistant Schizophrenia (m-RESIST). Clinical Trials. 2017. URL: https://ClinicalTrials.gov/ct2/show/

NCT03064776? cond=Study+of+m-RESIST\%2C + an + m-Health+Program + for + Treatment-resistant+Schizophrenia\&rank=1 [accessed 2018-11-27]

60. Development of a Mobile System for Self-Management of Schizophrenia (SOS). Clinical Trials. 2013. URL: https:/ /ClinicalTrials.gov/ct2/show/

NCT01969500?cond=Development+of+a+Mobile+System+for+Self-Management+of+Schizophrenia+\%28SOS\%29\&rank=1 [accessed 2018-11-27]

61. The Efficacy of Using a Smartphone App to Support Shared Decision Making in People With a Diagnosis of Schizophrenia. Clinical Trials. 2018. URL: https://ClinicalTrials.gov/ct2/show/NCT03554655 [accessed 2018-11-27]

62. MedActive: A Smartphone Intervention to Improve Adherence to Antipsychotic Medications. Clinical Trials. 2013. URL: https://ClinicalTrials.gov/ct2/show/

NCT01953237? cond=MedActive\%3A+A+Smartphone+Intervention+to+Improve+Adherence+to+AntiPsychotic+Medications\&rank=1 [accessed 2018-11-27]

63. A New Paradigm for Illness Monitoring and Relapse Prevention in Schizophrenia. Clinical Trials. 2013. URL: https:/ /ClinicalTrials.gov/ct2/show/

NCT01952041? cond=A+New+Paradigm+for+Illness+Monitoring+and+Relapse+Prevention+in+Schizophrenia\&rank=1 [accessed 2018-11-27]

64. Mobile Health Technology to Enhance Abstinence in Smokers With Schizophrenia. Clinical Trials. 2015. URL: https:/ /ClinicalTrials.gov/ct2/show/

NCT02420015? cond=Mobile+Health+Technology+to+Enhance+Abstinence+in+Smokers+with+Schizophrenia\&rank=1 [accessed 2018-11-27]

65. Real-Time Mobile Cognitive Behavioral Intervention for Serious Mental Illness. Clinical Trials. 2014. URL: https:/ /ClinicalTrials.gov/ct $2 /$ show/

NCT02035202? cond=Real-Time+Mobile+Cognitive+Behavioral+Intervention+for+Serious+Mental+Illness\&rank=1 [accessed 2018-11-27]

66. Feasibility and Outcomes of a Digital Health Support for the Schizophrenia Spectrum. Clinical Trials. 2018. URL: https:/ /ClinicalTrials.gov/ct2/show/

NCT03649815? cond=Feasibility+and+Outcomes+of+a+Digital+Health+Support+for+the+Schizophrenia+Spectrum\&rank=1 [accessed 2018-11-27]

67. Motivation and Skills Support (MASS). Clinical Trials. 2018. URL: https://ClinicalTrials.gov/ct2/show/ NCT03404219? cond=Motivation+and+Skills+Support+\%28MASS\%29\&rank=1 [accessed 2018-11-27]

68. Smartphone Addiction Recovery Coach for Young Adults (SARC-YA) Experiment (SARC-YA). Clinical Trials. 2017. URL: https://ClinicalTrials.gov/ct2/show/

NCT03301012? cond=Smartphone+Addiction+Recovery+Coach+for+Adolescents+\%28SARC-A\%29+Experiment\&rank=1 [accessed 2018-11-27]

69. The Cedar Project: Impact of mHealth for HIV Prevention Among Young Indigenous People Who Use Illicit Drugs. Clinical Trials. 2015. URL: https://ClinicalTrials.gov/ct2/show/NCT02437123 [accessed 2018-11-27]

70. Mobile Application to Improve Care Coordination Among HIV Clinic and Substance Use Treatment Providers. Clinical Trials. 2016. URL: https://ClinicalTrials.gov/ct2/show/ 
NCT02906215?cond=Mobile+Application+to+Improve+Care+Coordination+Among+HIV+Clinic+and+Substance+Use+Providers\&rank=1 [accessed 2018-11-27]

71. Mental Health Engagement Network (MHEN). Clinical Trials. 2011. URL: https://ClinicalTrials.gov/ct2/show/ NCT01473550?cond=Mental+Health+Engagement+Network+\%28MHEN\%29\&rank=1 [accessed 2018-11-27]

72. Mobile Health Cognitive Stimulation in Heroin Users (Re@ dict). Clinical Trials. 2014. URL: https://ClinicalTrials.gov/

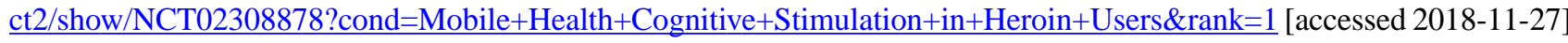

73. Automated Recovery Line for Medication Assisted Treatment. Clinical Trials. 2014. URL: https:/ClinicalTrials.gov/ct2/ show/NCT02124980?cond=Automated+Recovery+Line+for+Medication+Assisted+Treatment\&rank=1 [accessed 2018-11-27]

74. Homeless Care Management App (Link2Care). Clinical Trials. 2018. URL: https://ClinicalTrials.gov/ct2/show/ NCT03399500?term=NCT03399500\&rank=1 [accessed 2018-11-27]

75. Augmenting Specialty Eating Disorder Clinical Treatment With a Smartphone Application. Clinical Trials. 2015. URL: https://ClinicalTrials.gov/ct2/show/

NCT02484794?cond=Augmenting+Specialty+Eating+Disorder+Clinical+Treatment+with+a+Smartphone+Application\&rank=1 [accessed 2018-11-27]

76. Impact of Preanesthetic Information and Behavioral Intervention Using Smartphone on Anxiety of Children. Clinical Trials. 2014. URL: https://ClinicalTrials.gov/ct2/show/

NCT02246062?cond=Impact+of+Preanesthetic+Information+and+Behavioral+Intervention+Using+Smartphone+on+Anxiety+of+Children\&rank=1 [accessed 2018-11-27]

77. Effect of Premedication Type on Preoperative Anxiety in Children (EPPA). Clinical Trials. 2018. URL: https://ClinicalTrials. gov/ct2/show/NCT03530670? cond=Effect+of+Premedication+Type+on+Preoperative+Anxiety+in+Children\&rank=1 [accessed 2018-11-27]

78. Smartphone-Based Exposure Treatment for Dental Anxiety. Clinical Trials. 2018. URL: https://ClinicalTrials.gov/ct2/show/ NCT03461016? cond=Smartphone-Based+Exposure+Treatment+for+Dental+Anxiety\&rank=1 [accessed 2018-11-27]

79. Effects of Complementary Therapies Delivered Via Mobile Technologies. Clinical Trials. 2014. URL: https://ClinicalTrials. gov/ct2/show/NCT02236455? cond=Effects+of+Complementary+Therapies+Delivered+Via+Mobile+Technologies\&rank=1 [accessed 2018-11-27]

80. Using Smartphones to Enhance the Treatment of Childhood Anxiety (Smart-CAT). Clinical Trials. 2014. URL: https:/ /ClinicalTrials.gov/ct2/show/

NCT02259036?cond=Using+Smartphones+to+Enhance+the+Treatment+of+Childhood+Anxiety\&rank=1 [accessed 2018-11-27]

81. Youth Mayo Clinic Anxiety Coach Pilot Study. Clinical Trials. 2014. URL: https://ClinicalTrials.gov/ct2/show/NCT02205177 [accessed 2018-11-27]

82. ACT-smart: Smartphone-supplemented iCBT for Social Phobia and/or Panic Disorder. Clinical Trials. 2013. URL: https:/ /ClinicalTrials.gov/ct $2 /$ show/

NCT01963806? cond=ACT-smart\%3A+Smartphone-supplemented+iCBT+for+Social+Phobia+and\%2For+Panic+Disorder\&rank=1 [accessed 2018-11-27]

83. Evaluating the Psychophysiological Effects of a Smartphone-Based Mindfulness Task. Clinical Trials. 2017. URL: https:/ /ClinicalTrials.gov/ct2/show/

NCT03296007? cond=Evaluating+the+Psychophysiological+Effects+of+a+Smartphone-Based+Mindfulness+Task\&rank=1 [accessed 2018-11-27]

84. The Effectiveness of a Smartphone Application in the Treatment of Alcohol Use Disorder. Clinical Trials. 2018. URL: https://ClinicalTrials.gov/ct2/show/

NCT03396887? cond=The+Effectiveness+of+a+Smartphone+Application+in+the+Treatment+of+Alcohol+Use+Disorder\&rank=1 [accessed 2018-11-27]

85. AlcoChange: An Open Label Pilot Study of Smartphone Monitoring for Alcoholic Liver Disease. Clinical Trials. 2018. URL: https://ClinicalTrials.gov/ct2/show/ NCT03474328?cond=AlcoChange $\% 3 \mathrm{~A}+\mathrm{An}+$ Open+Label+Pilot+Study+of+Smartphone+Monitoring+for+Alcoholic+Liver+Disease\&rank=1 [accessed 2018-11-27]

86. Health Mobile Cognitive Stimulation in Alcoholics. Clinical Trials. 2013. URL: https://ClinicalTrials.gov/ct2/show/ NCT01942954?cond=Health+Mobile+Cognitive+Stimulation+in+Alcoholics\&rank=1 [accessed 2018-11-27]

87. Study of Mobile Phone Delivered Intervention to Reduce Alcohol Consumption (mROAD). Clinical Trials. 2014. URL: https://ClinicalTrials.gov/ct2/show/NCT02158949?term=NCT02158949\&rank=1 [accessed 2018-11-27]

88. Project Guard: Reducing Alcohol Misuse/Abuse in the National Guard. Clinical Trails. 2014. URL: https://ClinicalTrials. gov/ct2/show/

NCT02860442? cond=Project+Guard\%3A+Reducing+Alcohol+Misuse\%2FAbuse+in+the+National+Guard\&rank=1 [accessed 2018-11-27]

89. A Text Message Behavioral Intervention to Reduce Alcohol Consumption in Young Adults (TRAC). Clinical Trials. 2012. URL: https://ClinicalTrials.gov/ct2/show/NCT01688245?term=NCT01688245\&rank=1 [accessed 2018-12-27] 
90. A Tailored Physical Activity Smartphone App for Patients With Alcohol Dependence. Clinical Trials. 2016. URL: https:/ /ClinicalTrials.gov/ct2/show/

NCT02958280? cond=A+Tailored+Physical+Activity+Smartphone+App+for+Patients+With+Alcohol+Dependence \&rank=1 [accessed 2018-11-27]

91. Young Adult Naturalistic Alcohol Study (YANAS) Using Smartphone Technology in a Simulated Laboratory Environment. Clinical Trials. 2016. URL: https://ClinicalTrials.gov/ct2/show/NCT02841735?term=NCT02841735\&rank=1 [accessed 2018-11-27]

92. Smartphone Technology: Young Adult Drinking (STEADY). Clinical Trials. 2016. URL: https://ClinicalTrials.gov/ct2/ show/NCT02963818? cond=Smartphone+Technology\%3A+Young+Adult+Drinking+\%28STEADY\%29\&rank=1 [accessed 2018-11-27]

93. Smartphone-Paired Breathalyzers and Loss- and Gain-Framed Texts for Reducing Drinking and Driving (BESAFE). Clinical Trials. 2017. URL: https://ClinicalTrials.gov/ct2/show/NCT03335735?term=NCT03335735\&rank=1 [accessed 2018-11-27]

94. Adaptive Goal-Directed Adherence Tracking and Enhancement (AGATE). Clinical Trial. 2011. URL: https://ClinicalTrials. gov/ct2/show/NCT01349985?term=NCT01349985\&rank=1 [accessed 2018-11-27]

95. The Efficacy of A Smartphone-Based Support System to Reinforce Alcohol Abstinence in Treatment-Seeking Patients. Clinical Trials. 2014. URL: https://ClinicalTrials.gov/ct2/show/

NCT02385643?cond=The+Efficacy+of+A+Smartphone-based+Support+System+to+Reinforce+Alcohol+Abstinence+in+Treatment-seeking+Patients\&rank=1 [accessed 2018-11-27]

96. Usefulness of Supportive Text Messages in the Treatment of Depressed Alcoholics. Clinical Trials. 2009. URL: https:/ /ClinicalTrials.gov/ct2/show/

NCT01037868? cond=Usefulness+of+Supportive+Text+Messages+in+the+Treatment+of+Depressed+Alcoholics\&rank=1 [accessed 2018-11-27]

97. Text Messaging to Reduce Alcohol Relapse in Liver Transplant Patients. Clinical Trials. 2018. URL: https://ClinicalTrials. gov/ct2/show/NCT03402256?term=NCT03402256\&rank=1 [accessed 2018-11-27]

98. Lifestyle Physical Activity Intervention for Depressed Alcohol Dependent Women. Clinical Trials. 2016. URL: https:/ /ClinicalTrials.gov/ct2/show/

NCT02705898?cond=Lifestyle+Physical+Activity+Intervention+for+Depressed+Alcohol+Dependence\&rank=1 [accessed 2018-11-27]

99. Reducing Non-Medical Opioid Use: An Automatically Adaptive mHealth Intervention. Clinical Trials. 2016. URL: $\underline{\text { https:/ }}$ /ClinicalTrials.gov/ct $2 /$ show/

NCT02990377?cond=Reducing+Non-Medical+Opioid+Use\%3A+An+Automatically+Adaptive+mHealth+Intervention\&rank=1 [accessed 2018-11-27]

100. mHealth for Patient Self-Management of Opioid Use Disorder. Clinical Trials. 2018. URL: https://ClinicalTrials.gov/ct2/ show/NCT03633929? cond=mHealth+for+Patient+Self-Management+of+Opioid+Use+Disorder\&rank=1 [accessed 2018-11-27]

101. A Mobile Application for Post-Op Analgesic Consumption. Clinical Trials. 2017. URL: https://ClinicalTrials.gov/ct2/show/ NCT03197311?cond=A+Mobile+Application+for+Post-op+Analgesic+Consumption\&rank=1 [accessed 2018-11-27]

102. Using mHealth to Aid Opioid Medication Adherence Pilot Study. Clinical Trails. 2013. URL: https://ClinicalTrials.gov/ ct $2 /$ show/NCT02017041? cond=Using+mHealth+to+Aid+Opioid+Medication+Adherence\&rank=1 [accessed 2018-11-27]

103. Using m-Health Tools to Reduce the Misuse of Opioid Pain Relievers. Clinical Trials. 2017. URL: https://ClinicalTrials. gov/ct2/show/NCT03012087? cond=Using+m-Health+Tools+to+Reduce+the+Misuse+of+Opioid+Pain+Relievers\&rank=1 [accessed 2018-11-27]

104. Mobile Intervention for Young Opioid Users. Clinical Trials. 2017. URL: https://ClinicalTrials.gov/ct2/show/ NCT03610672? cond=Mobile+Intervention+for+Young+Opioid+Users\&rank=1 [accessed 2018-11-27]

105. Clinical Effect Size of an Educational Intervention in the Home and Compliance on People Who Suffer From Stroke. Clinical Trials. 2013. URL: https://ClinicalTrials.gov/ct2/show/NCT01980641 [accessed 2018-11-27]

106. The Adherence and Knowledge Exchange Heart and Stroke Medicines Study (TAKEmeds). Clinical Trials. 2015. URL: https://ClinicalTrials.gov/ct2/show/NCT02597205?cond=TAKEmeds\&rank=1 [accessed 2018-11-27]

107. My Stroke Team (MYST): Stroke App Pilot Study. Clinical Trials. 2014. URL: https://ClinicalTrials.gov/ct2/show/ NCT02230280?cond=myst+stroke\&rank=1 [accessed 2018-11-27]

108. iADAPT to Support Strategy Training After Stroke. Clinical Trials. 2017. URL: https://ClinicalTrials.gov/ct2/show/ NCT03253601?cond=iADAPTS+to+Support+Strategy+Training+After+Stroke\&rank=1 [accessed 2018-11-27]

109. Improving Medication Adherence Through SMS (Short Messaging Service) in Adult Stroke Patients: a Randomised Controlled Behaviour Intervention Trial. Clinical Trials. 2013. URL: https://ClinicalTrials.gov/ct2/show/NCT01986023 [accessed 2018-11-27]

110. TeleRehab for Stroke Patients Using Mobile Technology. Clinical Trials. 2015. URL: https://ClinicalTrials.gov/ct2/show/ NCT02615132?cond=telerehab+stroke\&rank=1 [accessed 2018-11-27]

111. Developing Accessible mHealth Programs for Depression Management in Bolivia. Clinical Trials. 2016. URL: https:/ /ClinicalTrials.gov/ct2/show/ 
NCT02765542? cond=Developing+Accessible+mHealth+Programs+for+Depression+Management+in+Bolivia\&rank=1 [accessed 2018-11-27]

112. mHealth for Antenatal Mental Health (AMHS). Clinical Trials. 2019. URL: https://ClinicalTrials.gov/ct2/show/ NCT02516982?cond=mHealth+for+Antenatal+Mental+Health\&rank=1 [accessed 2018-11-27]

113. Scaling Up Science-Based Mental Health Interventions in Latin America (DIADA). Clinical Trials. 2018. URL: https:/ /ClinicalTrials.gov/ct2/show/NCT03392883?term=NCT03392883\&rank=1 [accessed 2018-11-27]

114. Behavioural Activation-Based Treatment Administered Through Smartphone. Clinical Trials. 2011. URL: https:/ /ClinicalTrials.gov/ct2/show/

NCT01463020?cond=Behavioural+Activation-Based+Treatment+Administered+Through+Smartphone\&rank=1 [accessed 2018-11-27]

115. Mobile Sensing and Support for Depression (MOSS). Clinical Trials. 2016. URL: https://ClinicalTrials.gov/ct2/show/ NCT02776839?cond=Mobile+Sensing+and+Support+for+Depression\&rank=1 [accessed 2018-11-27]

116. Mobile Technology to Engage and Link Patients and Providers in Antidepressant Treatment (MedLink). Clinical Trials. 2015. URL: https://ClinicalTrials.gov/ct2/show/NCT02583230?term=NCT02583230\&rank=1 [accessed 2018-11-27]

117. Mobile Technology to Engage and Link Patients and Providers in Antidepressant Treatment (Medlink RCT). Clinical Trials. 2013. URL: https://ClinicalTrials.gov/ct2/show/

NCT01909973? cond=Mobile+Technology+to+Engage+and+Link+Patients+and+Providers+in+Antidepressant+Treatment\&rank=2 [accessed 2018-11-27]

118. Wellness Monitoring for Major Depressive Disorder (CBN-Well). Clinical Trials. 2016. URL: https://ClinicalTrials.gov/ ct2/show/NCT02934334?term=NCT02934334\&rank=1 [accessed 2018-11-27]

119. Patient Management of Depression Through Technology: A Study of Digitally Enabled Engagement. Clinical Trials. 2013. URL: https://ClinicalTrials.gov/ct2/show/

NCT03242213?cond=Patient+Management+of+Depression+Through+Technology\%3A+A+Study+of+Digitally+Enabled+Engagement\&rank=1 [accessed 2018-11-27]

120. Effectiveness of a Mobile Texting Intervention for People With Serious Mental Illness. Clinical Trials. 2017. URL: https:/ /ClinicalTrials.gov/ct2/show/

NCT03062267?cond=Effectiveness+of+a+Mobile+Texting+Intervention+for+People+With+Serious+Mental+Illness\&rank=1 [accessed 2018-11-27]

121. Psychotherapeutic Text Messaging for Depression Pilot Study (TEXT4U). Clinical Trials. 2016. URL: https://ClinicalTrials. gov/ct2/show/NCT02872454?cond=Psychotherapeutic+Text+Messaging+for+Depression+Pilot+Study\&rank=1 [accessed 2018-11-27]

122. Treating Depression on a Day-to-Day Basis: Development of a Tool for Physicians Based on a Smartphone Application (SMART). Clinical Trials. 2018. URL: https://ClinicalTrials.gov/ct2/show/

NCT03678194?cond=Treating+Depression+on+a+Day-today+Basis\%3A+Development+of+a+Tool+for+Physicians+Based+on+at+Smatphone+Application\&rank=1 [accessed 2018-11-27]

123. Testing the Value of Smartphone Assessments of People With Mood Disorders. Clinical Trials. 2018. URL: https:/ /ClinicalTrials.gov/ct2/show/

NCT03429361? cond=Testing+the+Value+of+Smartphone+Assessments+of+People+with+Mood+Disorders\&rank=1 [accessed 2018-11-27]

124. Evolution of Dark Ideas When Introducing or Switching an Antidepressant (DEPASSE). Clinical Trials. 2017. URL: https:/ /ClinicalTrials.gov/ct2/show/NCT03327974?term=NCT03327974\&rank=1 [accessed 2018-11-01]

125. IntelliCare: Artificial Intelligence in a Mobile Intervention for Depression and Anxiety (AIM). Clinical Trials. 2014. URL: https://ClinicalTrials.gov/ct $2 /$ show/

NCT02176226?cond=IntelliCare\%3A+Artificial+Intelligence+in+a+Mobile+Intervention+for+Depression+and+Anxiety+\%28AIM\%29\&rank=1 [accessed 2018-11-27]

126. Augmenting Hospitalization for Serious Mental Illness: Cognitive Bias Modification. Clinical Trials. 2018. URL: https:/ /ClinicalTrials.gov/ct $2 /$ show/

NCT03509181? cond=Augmenting+Hospitalization+for+Serious+Mental+Illness\%3A+Cognitive+Bias+Modification\&rank=1 [accessed 2018-11-27]

127. Evaluation of Text Message Engagement Support of Mindfulness Smartphone Applications. Clinical Trials. 2018. URL: https://ClinicalTrials.gov/ct2/show/

NCT03633682? cond=Evaluation+of+Text+Message+Engagement+Support+of+Mindfulness+Smartphone+Applications\&rank=1 [accessed 2018-11-27]

128. Enhancing Delivery of Problem Solving Therapy Using SmartPhone Technology. Clinical Trials. 2013. URL: https:/ /ClinicalTrials.gov/ct2/show/

NCT01891734?cond=Enhancing+Delivery+of+Problem+Solving+Therapy+Using+SmartPhone+Technology\&rank=1 [accessed 2018-11-27]

129. Mobile Phone Sensing and Outreach as Adjuncts to Internet-Based Behavioral Intervention for Depression. Clinical Trials. 2010. URL: https://ClinicalTrials.gov/ct2/show/NCT01107041?term=NCT01107041\&rank=1 [accessed 2018-11-27] 
130. Technology Enabled Mental Health Intervention for Individuals in the Criminal Justice System. Clinical Trials. 2017. URL: https://ClinicalTrials.gov/ct2/show/

NCT03105973? cond=Technology+Enabled+Mental+Health+Intervention+for+Individuals+in+the+Criminal+Justice+System\&rank=1 [accessed 2018-11-27]

131. Therapeutic Efficacy of Categorical Language Fluency Smartphone Game Application. Clinical Trials. 2016. URL: https:/ /ClinicalTrials.gov/ct2/show/

NCT02848404? cond=Therapeutic+Efficacy+of+Categorical+Language+Fluency+Smartphone+Game+Application\&rank=1 [accessed 2018-11-27]

132. Improving Medical Care With Electronic Interventions Based on Automated Text and Phone Messages. Clinical Trials. 2916. URL: https://ClinicalTrials.gov/ct2/show/

NCT03002311?cond=Improving+Medical+Care+With+Electronic+Interventions+Based+on+Automated+Text+and+Phone+Messages\&rank=1 [accessed 2018-11-27]

133. Youth Mayo Clinic Anxiety Coach Randomized Controlled Trial. Clinical Trials. 2014. URL: https://ClinicalTrials.gov/ ct $2 /$ show/NCT02205203? cond=Youth+Mayo+Clinic+Anxiety+Coach+Randomized+Controlled+Trial\&rank=1 [accessed 2018-11-27]

134. mWELLCARE:An Integrated mHealth System for the Prevention and Care of Chronic Disease (mWELLCARE). Clinical Trials. 2015. URL: https://ClinicalTrials.gov/ct2/show/NCT02480062 [accessed 2018-11-27]

135. Smartphone Based Continuing Care for Alcohol. Clinical Trials. 2016. URL: https://ClinicalTrials.gov/ct2/show/ NCT02681406? cond=Smartphone+Based+Continuing+Care+for+Alcohol\&rank=1 [accessed 2018-11-27]

136. Feasibility of a Smart-Phone Based Support System for Hazardous Drinkers (NZStepAway). Clinical Trials. 2018. URL: https://ClinicalTrials.gov/ct2/show/NCT03553056?term=NCT03553056\&rank=1 [accessed 2018-11-27]

137. Skills-Training for Reducing Risky Alcohol Use in App Form. Clinical Trials. 2018. URL: https://ClinicalTrials.gov/ct2/ show/NCT03696888? cond=Skills-Training+for+Reducing+Risky+Alcohol+Use+in+App+Form\&rank=1 [accessed 2018-11-27]

138. Impact on Opioid Use of Bundling Medication-assisted Treatment With mHealth (Bundling). Clinical Trials. 2016. URL: https://ClinicalTrials.gov/ct2/show/NCT02712034?cond=. +Impact+on+Opioid+Use+of+Bundling+Medication-assisted+Treatment+with+mHealth+\%28Bundling\%29\&rank=1 [accessed 2018-11-27]

139. Needle-X: Usability Testing of a Smartphone Application. Clinical Trials. 2018. URL: https:/ClinicalTrials.gov/ct2/show/ NCT03665298? cond=Needle-X\%3A+Usability+Testing+of+Smartphone+Application\&rank=1 [accessed 2018-11-27]

140. Using mHealth to Aid Opioid Addicts. Clinical Trials. 2013. URL: https://ClinicalTrials.gov/ct2/show/ NCT01955902?cond=Using+mHealth+to+Aid+Opioid+Addicts\&rank=1 [accessed 2018-11-27]

141. Smartphone Technology to Alleviate Malignant Pain (STAMP). Clinical Trials. 2018. URL: https://ClinicalTrials.gov/ct2/ show/NCT03717402? cond=Smartphone+Technology+to+Alleviate+Malignant+Pain+\%28STAMP\%29\&rank=1 [accessed 2018-11-27]

142. The Application of Wearable Technology to Improve the Physical Activity Level of People With Chronic Mental Illness. Clinical Trials. 2018. URL: https://ClinicalTrials.gov/ct2/show/ NCT03408327? cond=The+Application+of+Wearable+Technology+to+Improve+the+Physical+Activity+Level+of+People+with+Chronic+Mental+Illness\&rank=1 [accessed 2018-11-27]

143. Feasibility and Acceptability of a Smartphone App to Assess Early Warning Signs of Psychosis Relapse (ExPRESS:2). Clinical Trials. 2018. URL: https://ClinicalTrials.gov/ct2/show/NCT03558529 [accessed 2018-11-27]

144. Using Mobile Technology to Enhance Early Psychosis Treatment Delivery (RWJFGinger). Clinical Trials. 2017. URL: https://ClinicalTrials.gov/ct2/show/

NCT03303456? cond=Using+Mobile+Technology+to+Enhance+Early+Psychosis+Treatment+Delivery\&rank=1 [accessed 2018-11-27]

145. Smartphone Applications Youth With Early Psychosis in Community Outpatient Settings (BHCOEMobi). Clinical Trials. 2017. URL: https://ClinicalTrials.gov/ct2/show/

NCT03286595?cond=Smartphone+Applications+Youth+with+Early+Psychosis+in+Community+Outpatient+Settings\&rank=1 [accessed 2018-11-27]

146. Mobile Enhancement of Motivation in Schizophrenia. Clinical Trials. 2017. URL: https://ClinicalTrials.gov/ct2/show/ NCT03059771?cond=Mobile+Enhancement+of+Motivation+in+Schizophrenia\&rank=1 [accessed 2018-11-27]

147. Waitlist-Control Trial of Smartphone CBT for Body Dysmorphic Disorder (BDD). Clinical Trials. 2018. URL: https:/ /ClinicalTrials.gov/ct2/show/

NCT03673046? cond=Waitlist-Control+Trial+of+Smartphone+CBT+for+Body+Dysmorphic+Disorder+\%28BDD\%29\&rank=1 [accessed 2018-11-27]

148. Preventing HIV/STI in Urban Adolescents Via an mHealth Primary Care Intervention. Clinical Trials. 2017. URL: https:/ /ClinicalTrials.gov/ct2/show/

NCT03368456? cond=Preventing+HIV\%2FSTI+in+Urban+Adolescents+via+an+mHealth+Primary+Care+Intervention\&rank=1 [accessed 2018-11-27] 
149. CopeSmart: Using Mobile Technology to Promote Positive Mental Health in Young People. Clinical Trials. 2014. URL: https://ClinicalTrials.gov/ct2/show/

NCT02265978?cond=CopeSmart\%3A+Using+Mobile+Technology+to+Promote+Positive+Mental+Health+In+Young+People\&rank=1 [accessed 2018-11-27]

150. Tse T, Fain KM, Zarin DA. How to avoid common problems when using ClinicalTrials.gov in research: 10 issues to consider. Br Med J 2018 May 25;361:k1452 [FREE Full text] [doi: 10.1136/bmj.k1452] [Medline: 29802130]

151. Williams RJ, Tse T, Harlan WR, Zarin DA. Registration of observational studies: is it time? Can Med Assoc J 2010 Oct 19;182(15):1638-1642 [FREE Full text] [doi: 10.1503/cmaj.092252] [Medline: 20643833]

152. Effects of Patient-Centered Stroke Educating System: A Randomized Controlled Trial. Clinical Trials. 2018. URL: https:/ /ClinicalTrials.gov/ct2/show/NCT02591511?cond=stroke+educating+system\&rank=1 [accessed 2018-11-27]

153. mWELLCARE:An Integrated mHealth System for the Prevention and Care of Chronic Disease (mWELLCARE). Clinical Trials. 2015. URL: https://ClinicalTrials.gov/ct2/show/NCT02480062 [accessed 2018-11-27]

154. Rayhill ML, Sharon R, Burch R, Loder E. Registration status and outcome reporting of trials published in core headache medicine journals. Neurology 2015 Nov 17;85(20):1789-1794. [doi: 10.1212/WNL.0000000000002127] [Medline: 26475691]

155. Safavi K, Mathews SC, Bates DW, Dorsey ER, Cohen AB. Top-funded digital health companies and their impact on high-burden, high-cost conditions. Health Aff (Millwood) 2019 Jan;38(1):115-123. [doi: 10.1377/hlthaff.2018.05081] [Medline: $\underline{30615535]}$

156. Calvo RA, Dinakar K, Picard R, Christensen H, Torous J. Toward impactful collaborations on computing and mental health. J Med Internet Res 2018 Feb 9;20(2):e49 [FREE Full text] [doi: 10.2196/jmir.9021] [Medline: 29426812]

157. Al-Durra M, Nolan RP, Seto E, Cafazzo JA, Eysenbach G. Nonpublication rates and characteristics of registered randomized clinical trials in digital health: cross-sectional analysis. J Med Internet Res 2018 Dec 18;20(12):e11924 [FREE Full text] [doi: 10.2196/11924] [Medline: 30485832]

158. Antes G, Chalmers I. Under-reporting of clinical trials is unethical. Lancet 2003 Mar 22;361(9362):978-979. [doi: 10.1016/S0140-6736(03)12838-3] [Medline: 12660049]

159. Hopewell S, Loudon K, Clarke M, Oxman A, Dickersin K. Publication bias in clinical trials due to statistical significance or direction of trial results. Cochrane Library 2009:-. [doi: 10.1002/14651858.mr000006.pub3]

160. Tfelt-Hansen PC. Unpublished clinical trials with sumatriptan. Lancet 2009 Oct 31;374(9700):1501-1502. [doi: 10.1016/S0140-6736(09)61906-1] [Medline: 19880018]

161. Gabapentin for Pain: New Evidence From Hidden Data. Evidence-Based Prescription Drug Therapy Information. 2009. URL: https://www.ti.ubc.ca/2009/12/31/gabapentin-for-pain-new-evidence-from-hidden-data/ [accessed 2019-01-05]

162. Kelders SM, Kok RN, Ossebaard HC, van Gemert-Pijnen JE. Persuasive system design does matter: a systematic review of adherence to web-based interventions. J Med Internet Res 2012 Nov 14;14(6):e152 [FREE Full text] [doi: 10.2196/jmir.2104] [Medline: 23151820]

163. Lee K, Bacchetti P, Sim I. Publication of clinical trials supporting successful new drug applications: a literature analysis. PLoS Med 2008 Sep 23;5(9):e191 [FREE Full text] [doi: 10.1371/journal.pmed.0050191] [Medline: 18816163]

164. Azar M, Riehm KE, McKay D, Thombs BD. Transparency of outcome reporting and trial registration of randomized controlled trials published in the journal of consulting and clinical psychology. PLoS One 2015;10(11):e0142894 [FREE Full text] [doi: 10.1371/journal.pone.0142894] [Medline: 26581079]

165. Barnett I, Torous J, Staples P, Keshavan M, Onnela J. Beyond smartphones and sensors: choosing appropriate statistical methods for the analysis of longitudinal data. J Am Med Inform Assoc 2018 Dec 1;25(12):1669-1674 [FREE Full text] [doi: 10.1093/jamia/ocy121] [Medline: $\underline{\text { 30272176] }}$

\author{
Abbreviations \\ DALYs: disability-adjusted life years \\ EU: European Union \\ ICJME: International Committee of Journal Medical Editors \\ IRB: Institutional Review Board \\ mHealth: mobile health \\ NIH: National Institutes of Health \\ NYU: New York University \\ SOM: School of Medicine \\ WHO: World Health Organization
}


Edited by $G$ Eysenbach; submitted 07.09.19; peer-reviewed by L Beattie, E Ding; comments to author 04.11.19; revised version received 21.11.19; accepted 26.01.20; published 04.08.20

Please cite as:

Minen MT, Reichel JF, Pemmireddy P, Loder E, Torous J

Characteristics of Neuropsychiatric Mobile Health Trials: Cross-Sectional Analysis of Studies Registered on ClinicalTrials.gov

JMIR Mhealth Uhealth 2020;8(8):e16180

URL: https://mhealth.jmir.org/2020/8/e16180

doi: $\underline{10.2196 / 16180}$

PMID: $\underline{32749230}$

CMia Tova Minen, Julia Frederica Reichel, Pallavi Pemmireddy, Elizabeth Loder, John Torous. Originally published in JMIR mHealth and uHealth (http://mhealth.jmir.org), 04.08.2020. This is an open-access article distributed under the terms of the Creative Commons Attribution License (https://creativecommons.org/licenses/by/4.0/), which permits unrestricted use, distribution, and reproduction in any medium, provided the original work, first published in JMIR mHealth and uHealth, is properly cited. The complete bibliographic information, a link to the original publication on http://mhealth.jmir.org/, as well as this copyright and license information must be included. 